\title{
A FORMULATION OF PEACEMAN AND RACHFORD ADI METHOD FOR THE THREE-DIMENSIONAL HEAT DIFFUSION EQUATION
}

\author{
I. A. Ismail *, E. H. Zahran **, and M. Shehata*** \\ * Dean of the Faculty of Computers \& Informatics Zagazig University. \\ ** Faculty of Engineering, Shobra, , Zagazig University, Banha branch \\ ***Department of Math. Faculty of Science Zagazig University, \\ Maha_Shehata@hotmail.com
}

\begin{abstract}
A formulation of an alternating direction implicit (ADI) method is given by extending peaceman and Rachford Scheme to three dimensions. The scheme becomes conditionally stable. The von Neumann stability analysis is performed. Numerical results for solving heat diffusion equation have been obtained for different specified boundary value problems to obtain a simple explicit stability.
\end{abstract}

Key Words- Heat-diffusion PDE, Boundary Value Problems, Von Neumann Stability Analysis, Numerical Methods, Finite Differences

\section{INTRODUCTION}

The alternating direction implicit (ADI) method was first introduced by peaceman and Rachford [1] in 1955 for two dimensional parabolic and elliptic partial differential equations. The theoretical aspects of the method were discussed by Douglas [2]. The original research included applying (ADI) technique to two dimensional solidification presented by Ananthanarayanan and Lahiri [4], using the peaceman and Rachford (ADI) method solving initial boundary value problems for partial differential equations in more than 2-D has been elaborated by Hundsdorfer and verwer [5], and the formulation of a peace man and Rachford (ADI) scheme in a region of arbitrary geometry done by shukla and Ghosh [6].

(N.B) All the software used in this work has been designed and written by the authors and could be demonstrated by them when required.

\section{NUMERICAL FORMULATION}

A linear partial differential equation for 3- dimensional diffusion problem in cartesian coordinate [7]:

$$
\frac{\partial u}{\partial t}=\frac{\partial^{2} u}{\partial x^{2}}+\frac{\partial^{2} u}{\partial y^{2}}+\frac{\partial^{2} u}{\partial z^{2}}
$$

Now split the diffusion terms on the right hand side of equation (1) and alternatively let each spatial direction be represented implicity while others explicity at (1/3) of time interval. This leads to a 3 - step scheme. The general form of equation (1) is 


$$
\begin{aligned}
& \frac{u_{l j k}^{n+1}-u_{l j k}^{n}}{\Delta t / 3}= \\
& {\left[\frac{u_{l+1, j, k}-2 u_{l j k}+u_{l-1, j, k}}{\Delta x^{2}}+\frac{u_{l, j+1, k}-2 u_{l, j, k}+u_{l, j-1, k}}{\Delta y^{2}}+\frac{u_{l, j, k-1}-2 u_{l, j, k}+u_{l, j, k+1}}{\Delta z^{2}}\right]^{n}} \\
& \frac{u_{l, j, k}^{n+1}-u_{l, j, k}^{n}}{\Delta t / 3}=\sum_{i=1}^{3} \frac{\delta_{\eta_{i}}^{2} u_{l, j, k}^{n}}{\Delta \eta_{i}^{2}}
\end{aligned}
$$

using ADI method, we get the following 3 - step scheme : step (1)

$$
\frac{u_{l, j, k}^{(1)}-u_{l, j, k}^{n}}{\Delta t / 3}=\frac{\delta_{\eta_{l}}^{2} u_{l, j, k}^{(1)}}{\Delta \eta_{i}^{2}}+\sum_{i=2}^{3} \frac{\delta_{\eta_{i}}^{2} u_{l, j, k}^{n}}{\Delta \eta_{i}^{2}}
$$

at step j :

$$
\frac{u_{l, j, k}^{j}-u_{l, j, k}^{j-1}}{\Delta t / 3}=\frac{\delta_{\eta_{j}}^{2} u_{l, j, k}^{j}}{\Delta \eta_{j}^{2}}+\sum_{\substack{i=1 \\ i \neq j}}^{3} \frac{\delta_{\eta_{i}}^{2} u_{l, j, k}^{j-1}}{\Delta \eta_{i}^{2}} \quad \text { where }(\mathrm{j}=2)
$$

and at step 3 :

$$
\frac{u_{l, j, k}^{n+1}-u_{i, j, k}^{2}}{\Delta t / 3}=\frac{\delta_{\eta_{3}}^{2} u_{l, j, k}^{n+1}}{\Delta \eta_{3}^{2}}+\sum_{i=1}^{3} \frac{\delta_{\eta_{i}}^{2} u_{l, j, k}^{2}}{\Delta \eta_{i}^{2}}
$$

where $\mathrm{u}^{(\mathrm{j})},(\mathrm{j}=1,2)$ are the intermediate temperature values associated with $j^{\text {th }}$ step and with $\mathrm{u}^{(0)}=\mathrm{u}^{(\mathrm{n})}$ and $\mathrm{u}^{(\mathrm{n}+1)}=\mathrm{u}^{(3)}$ where $\mathrm{n}$ and $\mathrm{n}+1$ denote two consecutive time levels. We define the numbers $r_{i}=\frac{\Delta t}{\Delta \eta_{i}^{2}}(i=1,2,3)$ and replace $\delta^{2}$ with central difference representations of second derivatives Eqs. (4), (5) and (6) can be rearranged as

Step 1 :

$$
-r_{1} u_{l-1, j, k}^{(1)}+\left(3+2 r_{1}\right) u_{l, j, k}^{1}-r_{1} u_{l+1, j, k}^{(1)}=\left(3-2 \sum_{i=2}^{3} r_{i}\right) u_{l, j, k}^{n}+\sum_{i=2}^{3} r_{i}\left(u_{l, j-1, k}^{n}+u_{l, j+1, k}^{n}\right)
$$

at step j :

$$
-r_{j} u_{l, j-1, k}^{(j)}+\left(3+2 r_{j}\right) u_{l, j, k}^{j}-r_{j} u_{l, j+1, k}^{(j)}=\left(3-2 \sum_{\substack{i=1 \\ i \neq j}}^{3} r_{i}\right) u_{l, j, k}^{j-1}+\sum_{\substack{i=1 \\ i \neq j}}^{3} r_{i}\left(u_{l, j-1, k}^{j-1}+u_{l, j+1, k}^{j-1}\right), j=2
$$

and at step 3 :

$$
-r_{3} u_{l, j, k-1}^{(n+1)}+\left(3+2 r_{3}\right) u_{l, j, k}^{n+1}-r_{3} u_{l, j, k+1}^{(j-1)}=\left(3-2 \sum_{i=1}^{2} r_{i}\right) u_{l, j, k}^{2}+\sum_{i=1}^{2} r_{i}\left(u_{l, j-1, k}^{2}+u_{l, j+1, k}^{2}\right)
$$


In these equations, all implicit terms with unknown temperature values are on here the left hand side, while all the explicit terms with known temperature values are on right hand side. Notic that Eqs. (7.9) are for the internal temperature nodes. We can obtain 3 matrix equations each of which consists of $\prod_{i=1}^{3} m_{i}$. Linear algebraic equations, where $m_{i}$ is the number of unknown temperature nodes in $j^{\text {th }}$ space :

$$
\begin{aligned}
& {\left[D_{1}\right]\left\{u^{(1)}\right\}=\left\{c_{1}\right\},} \\
& {\left[D_{j}\right]\left\{u^{(j)}\right\}=\left\{c_{j}\right\}, \quad j=2,}
\end{aligned}
$$

and

$$
\left[D_{3}\right]\left\{u^{n+1}\right\}=\left\{c_{3}\right\}
$$

The coefficients on the left hand side of Eqs. (7.9) form the tridiagonal elements of $\left[D_{j}\right],(j=1,2,3)$, respectively, while the terms on the right hand side of those equations form the vectors $\left\{c_{j}\right\}(j=1,2,3)$, respectively. Each of these equations can be solved in a rather straightforward manner by usingthe so called tridiagonal matrix algorithm (TDMA).

\section{STABILITY ANALYSIS}

The von Neumann stability analysis is perhaps the most widely used procedure for establishing the stability characteristics of a finite difference Scheme [3]. At a certain time step, the error takes the form.

$$
E_{j-1}=\exp \left[a\left(n+\frac{j-1}{3}\right) \Delta t\right] \exp \left(I \beta_{1} l \Delta \eta_{1}\right) \exp \left(I \beta_{2} j \Delta \eta_{2}\right) \exp \left(I \beta_{3} \cdot k \Delta \eta_{3 i}\right),(j=1,2,3)
$$

where $I=\sqrt{-1}$ and $\beta$ is the wave number. Since the error $\mathrm{E}$ satisfies the same difference equation as the exact numerical solution, substituting Eq. (13) into Eqs. (79) gives the amplification factors.

Then, the overall

$$
F_{j}=\frac{E_{j}}{E_{j=1}}=\exp \left(a \frac{\Delta t}{3}\right)=\frac{3-4 \sum_{i=1, i \neq j}^{3} r_{i} \sin ^{2}\left(\frac{\beta_{i} \Delta \eta_{i}}{2}\right)}{3+4 r_{j} \sin ^{2}\left(\frac{\beta_{j} \Delta \eta_{j}}{2}\right)}, \quad(j=1,2,3)
$$


The error will not grow if

$$
F_{j}=\prod_{j=1}^{3} F_{j}=\prod_{j=1}^{3} \frac{3-4 \sum_{i=1}^{3} r_{i} \sin ^{2}\left(\frac{\beta_{i} \Delta \eta_{i}}{2}\right)}{3+4 r_{j} \sin ^{2}\left(\frac{\beta_{j} \Delta \eta_{j}}{2}\right)}
$$

$$
|F| \leq 1
$$

this is known as the von Neumann condition. Rearranging the numerators in Eq. (13) so that no denominttor in the equation owns its original numerator, we obtain

$$
F=\prod_{j=1}^{3} \frac{3-4 \sum_{i=1}^{3} r_{i} \sin ^{2}\left(\frac{\beta_{i} \Delta \eta_{i}}{2}\right)}{3+4 r_{j} \sin ^{2}\left(\frac{\beta_{j} \Delta \eta_{j}}{2}\right)}=\prod_{j=1}^{3} F_{j}^{*}
$$

The von Neumann condition is satisfied if the absolute value of each of the modified amplification factors, $\mathrm{F}^{*}$, in $\mathrm{Eq}$. (17) is not greater than unity.

$$
\left|F_{j}^{*}\right| \leq 1 \quad(\mathrm{j}=1,2,3,)
$$

since $F_{j}^{*} \leq 1$ due to $\mathrm{r}_{\mathrm{i}}>0$, and $\mathrm{r}_{\mathrm{j}}>0(\mathrm{i}, \mathrm{j}=1,2,3)$, we consider only the case of $F_{j}^{*} \leq-1$ from Eq. (17).

$$
\frac{3-4 \sum_{i=1}^{3} r_{i} \sin ^{2}\left(\frac{\beta_{i} \Delta \eta_{i}}{2}\right)}{3+4 r_{j} \sin ^{2}\left(\frac{\beta_{j} \Delta \eta_{j}}{2}\right)} \geq-1, \quad(j=1,2,3)
$$

or

$$
\sum_{\substack{i=1 \\ i \neq j}}^{3} r_{i} \sin ^{2}\left(\frac{\beta_{i} \Delta \eta_{i}}{2}\right) \leq \frac{3}{2}, \quad(j=1,2,3)
$$

this is the stability requirement for the ADI scheme. Let all the terms of $\sin ^{2}\left(\frac{\beta_{i} \Delta \eta_{i}}{2}\right)$ equal unity. Eq. (19) becomes

$$
\sum_{\substack{i=1 \\ i \neq j}}^{3} r_{i} \leq 3 / 2 \quad \text { or } \quad \sum_{\substack{i=1 \\ i \neq j}}^{3} \frac{\Delta t}{\Delta \eta_{i}^{2}} \leq 3 / 2, \quad j=1,2,3
$$

let, $\mathrm{r}=\max (\mathrm{r} 1, \mathrm{r} 2, \mathrm{r} 3)$. Eq. (20) is satisfied if

$$
r \leq 3 / 2 \quad \text { or } \frac{\Delta t}{\Delta \eta^{2}} \leq 3 / 2
$$


Where $\Delta \eta=\max \left(\Delta \eta_{1}, \Delta \eta_{2}, \Delta \eta_{3}\right)$. This indicates that peaceman and Rachford ADI scheme becomes conditionally stable for 3 .dimensions, the stability constrain is 1.5 .

\section{APPLICATION AND DISCUSSION}

5 .

We shall introduce here a comparison between the numerical solutions of three different problems and its known analytical solutions.

In the first example, we solve the heat diffusion equation in the cube subject to homogeneous Dirichlet boundary conditions. Figure (1) shows the numerical solution of this example, which has analytical solution $u(x, y, z, t)=\sin (x \pi) \sin (y \pi) \sin (z \pi) \exp \left(-3 \pi^{2} t\right)$. We solve, throughout the second example, the heat diffusion equation in the cube subject to homogeneous Dirichlet boundary conditions. Figure (2) shows the numerical solution of this example, which has analytical solution $u(x, y, z, t)=\cos \left(\frac{\pi}{2} x\right) \cos \left(\frac{\pi}{2} y\right) \cos \left(\frac{\pi}{2} z\right) \exp \left(-3 \frac{\pi^{2}}{4} t\right)$.

In the final example, we solve the heat diffusion equation in the cube subject to homogeneous Dirichlet boundary conditions. Figure (3) shows the numerical solution of this example, which has analytical solution $u(x, y, z, t)=\left(1-x^{2}\right)\left(1-y^{2}\right)\left(1-z^{2}\right) \exp (-3 t)$

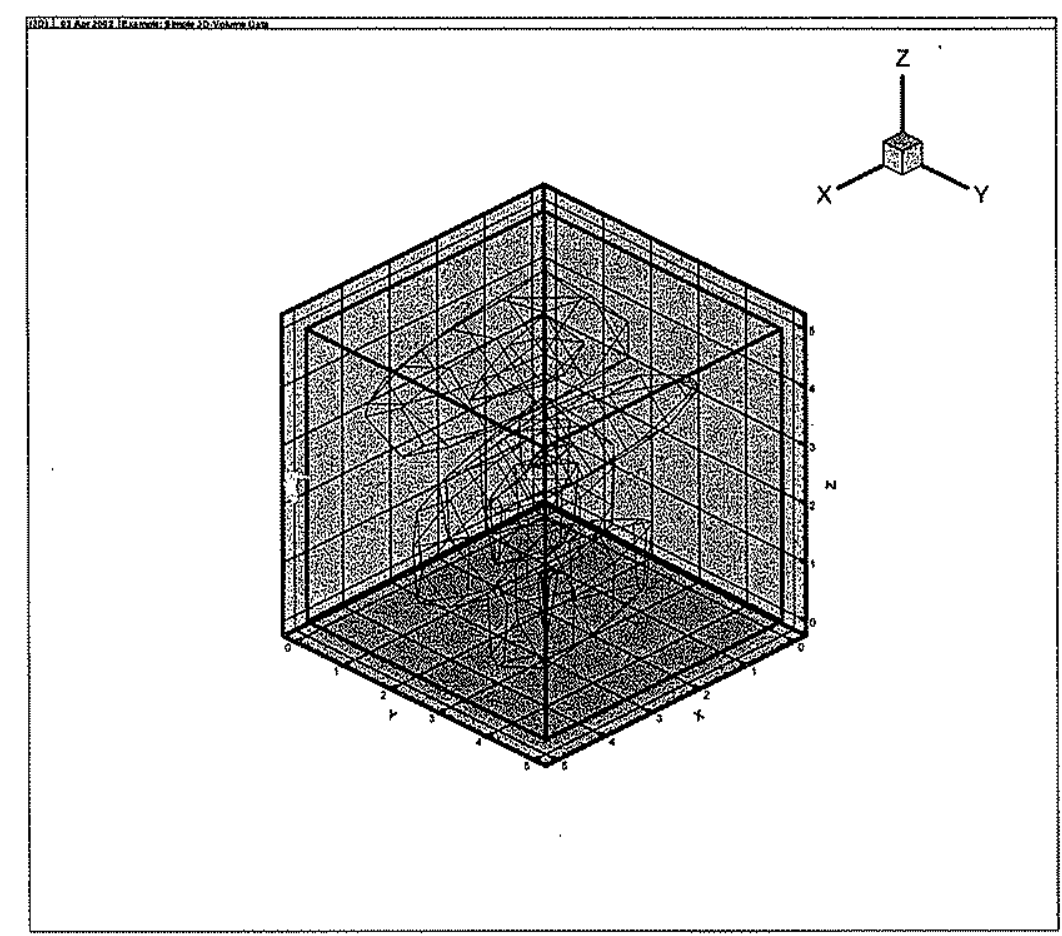

Fig. (1) 


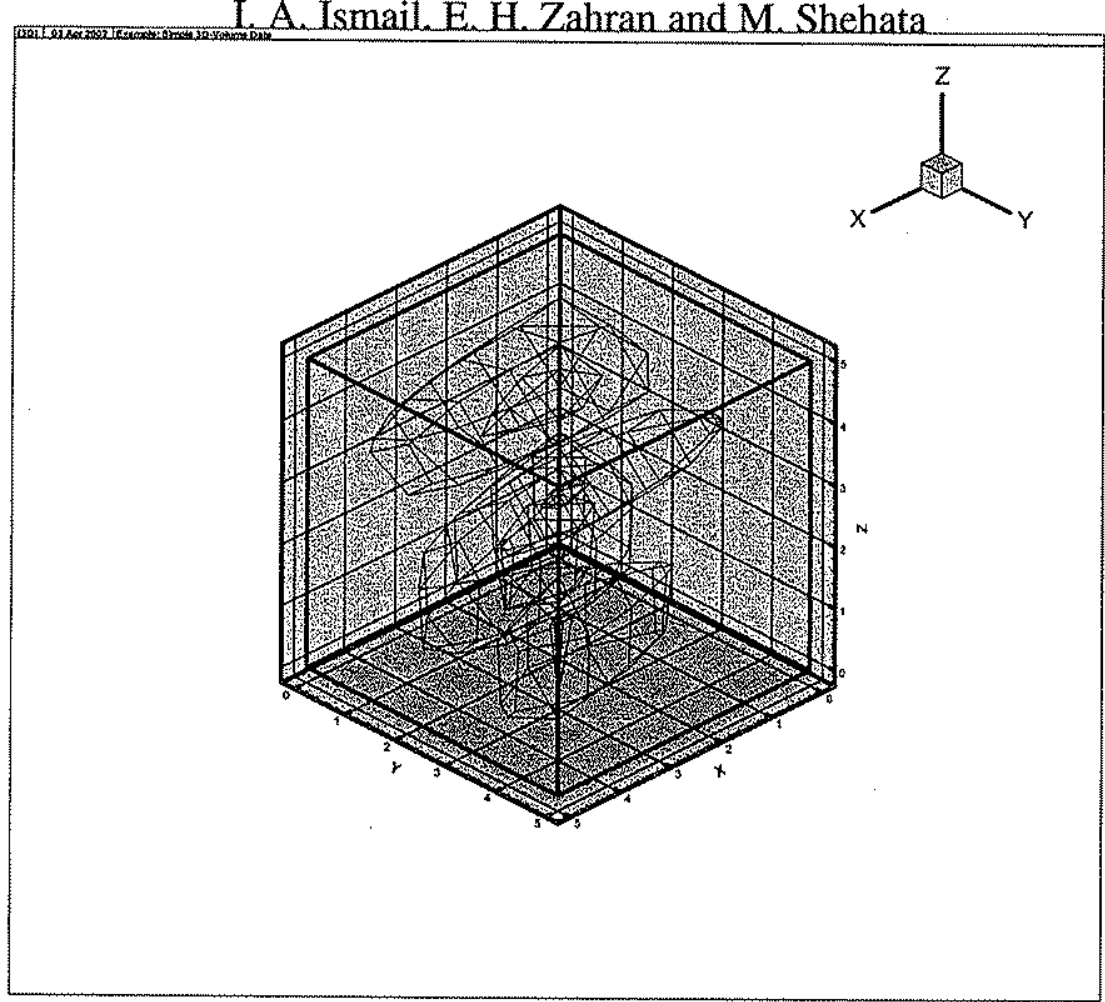

Fig. (2)

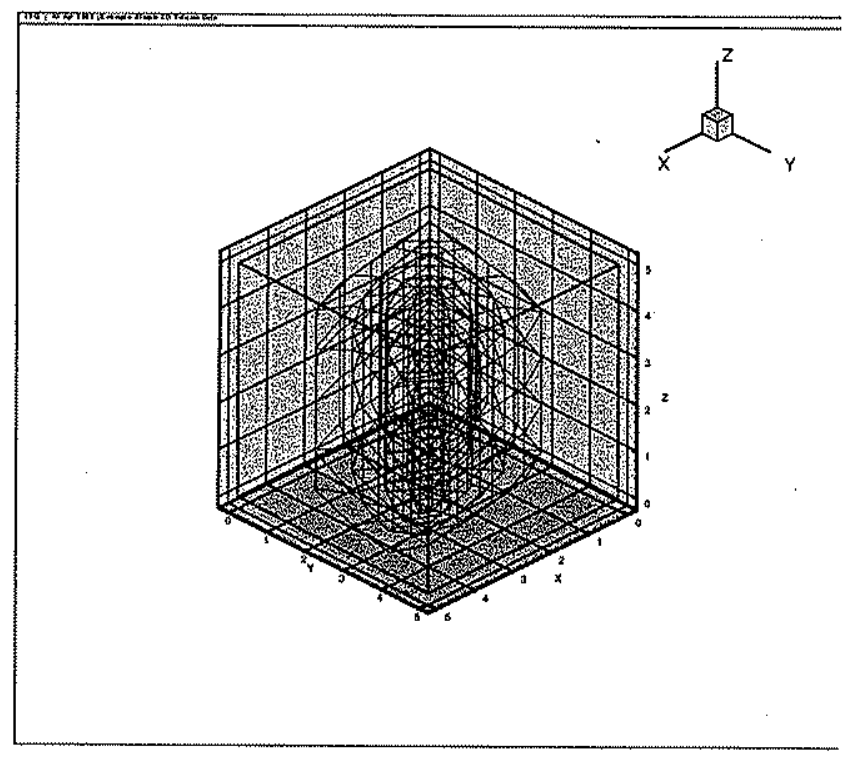

Fig. (3) 


\section{REFERENCES}

1. D. W. Peaceman and H.H Rachford, Jr. J Soc. Indust. Appl. Math., 3, p.28, 1955.

2. Jim. Douglas, Jr., J Soc. Indust. Appl. Math.,3, p.42, 1955.

3. W. J. Minkowycz, E. M. Sparrow, G. E. Schneider and R. H. Pletcher, Handbook of Numerical Heat Transfer, pp. 17-78, John Wiley \& sons Inc., New York 1988.

4. L. Ananthanarayanan and A.K. Lahiri, Int. Comm. Heat Mass Transfer, 19, p.629, 1992.

5. W.H. Hundsdorfer and J.G.Verwer, Mathematics of computation, $\mathbf{5 3}_{\mathbf{2}}$ p. 81 , 1989.

6. K.N. Shukla and D. Ghosh, Heat transfer Engineering, 15, p.64, 1994.

7. M. K. Jain "Numerical Solution of Differential Equations", Wiley Eastern Limited, New Delhi, 1984. 
